\title{
SUBGROUPS OF POLYNOMIAL AUTOMORPHISMS
}

\author{
EDWIN CONNELL AND JOHN ZWEIBEL
}

\section{INTRODUCTION}

Throughout this paper, $k$ will denote a commutative ring containing the rational numbers $\mathbf{Q}$, and $k^{[n]}=k\left[x_{1}, \ldots, x_{n}\right]$ will be the polynomial ring over $k$. If $f: k^{[n]} \rightarrow k^{[n]}$ is a polynomial map (i.e., a $k$-algebra homomorphism), then $f$ is a polynomial automorphism provided there is an inverse $f^{-1}$ which is also a polynomial map. Very little is known about the group of polynomial automorphisms, and indeed it is difficult to determine which polynomial maps are automorphisms. The purpose of this paper is to define a collection $H$ of polynomial maps, to show that each $h \in H$ is a polynomial automorphism and that $H$ is a group under composition, and to classify $H$ up to group isomorphism. As a consequence, this will prove a special case of the Jacobian conjecture.

If $V$ is an $n$-dimensional column vector over $k^{[n]}$ whose $i$ th term is $v_{i}$, then the Jacobian $J(V)$ is defined to be the $n \times n$ matrix $\left(\partial v_{i} / \partial x_{j}\right)$. Let $X$ denote the vector whose $i$ th term is $x_{i}$. If $g: k^{[n]} \rightarrow k^{[n]}$ is a polynomial map, then $g(X)$ will denote the vector whose $i$ th term is $g\left(x_{i}\right)$. This gives a bijection from polynomial maps to vectors. The Jacobian $J(g)$ of a polynomial map $g$ is defined to be the Jacobian of the vector $g(X)$. Suppose for the moment that $k$ is a field of characteristic 0 . The Jacobian conjecture states that if $f: k^{[n]} \rightarrow k^{[n]}$ is a polynomial map with $|J(f)|=1$, then $f$ is a polynomial automorphism. It is known that this conjecture can be reduced to the case where $J(f)=I+M$, where each term of the matrix $M$ is a homogeneous polynomial of degree 2 [BCW]. It follows from the theorem below that the Jacobian conjecture is true, provided it can be reduced to the case where $J(f)^{-1}=I-N$, where each term of $N$ is homogeneous of some fixed degree $q \geq 1$.

Received by the editors May 31, 1989 and, in revised form, February 4, 1990.

1980 Mathematics Subject Classification (1985 Revision). Primary 13B10, 13B25, $17 \mathrm{~B} 70$. 
Suppose $q \geq 1$ and $f: k^{[n]} \rightarrow k^{[n]}$ is a polynomial map with $f(\mathbf{0})=\mathbf{0}$. Suppose the Jacobian matrix has $|J(f)|=1$ and $J(f)^{-1}=I-N$ where $N \neq 0$ and each term of $N$ is a homogeneous polynomial of degree $q$. Then $N$ is nilpotent, i.e., there exists $r \geq 1$ so that $N^{r+1}=\mathbf{0}, N^{r} \neq \mathbf{0}$, and $J(f)=$ $I+N+\cdots+N^{r}$. An $n \times n$ matrix $M$ over $k^{[n]}$ is said to be exact provided there exists a vector $V$ with $J(V)=M$. In this case where $J(f)=I+N+\cdots+N^{r}$, each $N^{j}$ is exact. Let $H$ be the collection of all polynomial maps $h$ with $h(\mathbf{0})=\mathbf{0}$ and $J(h)=I+c_{1} N+\cdots+c_{r} N^{r}$ where each $c_{i} \in k$. The following theorem is taken from part (ii) of the main theorem below.

Theorem. Each $h$ in $H$ is a polynomial automorphism. $H$ is a subgroup of the group of all polynomial automorphisms on $k^{[n]}$.

Furthermore, it will be shown that, up to group isomorphism, $H$ depends only on $q$ and $r$, i.e., it does not depend on $n$ or the choice of $N$. In fact, for a fixed $q, H$ is isomorphic to a subgroup $G_{r}$ of automorphisms of a truncated polynomial ring in one variable. Thus this gives a representation of $G_{r}$ into the group of polynomial automorphisms on $k^{[n]}$. (It is unclear what conditions imply that two such representations are algebraically equivalent.)

Question. Suppose $H$ is as above, where $k$ is a field of characteristic 0 . Does there exist a nonzero linear form $l=a_{1} x_{1}+\cdots+a_{n} x_{n}$ such that $h(l)=l$ for all $h \in H$ ? This is equivalent to asking if the rows of $N$ are linearly dependent over $k$. More generally, does there exist a linear automorphism $L: k^{[n]} \rightarrow k^{[n]}$ such that $J\left(L h L^{-1}\right)$ is upper triangular for all $h \in H$ ?

We conclude the introduction with a special case of part (iii) of the main theorem. Suppose $g: k^{[n]} \rightarrow k^{[n]}$ is a polynomial automorphism with $J(g)=I+M$ and $M^{2}=0$. In general, it will not be true that $J\left(g^{2}\right)=J(g)^{2}$, i.e., it will not be true that $\left.J(g)\right|_{g}=J(g)$. However, in the case that there is $t \geq 1$ so that each term of $M$ is homogeneous of degree $t$, then $J\left(g^{2}\right)=J(g)^{2}$.

The main theorem and a sketch of the proof are presented below. The proper environment for this theory is not polynomial rings, but rather power series rings. In fact, the proof depends upon an exponential map which requires this environment. 


\section{THE MAIN THEOREM}

Let $k^{[n]]}$ be the formal power series ring over $k$, and let $I \subset$ $k^{[[n]]}$ be the augmentation ideal generated by the variables $\left\{x_{1}, \ldots, x_{n}\right\}$. Denote by $\widehat{G A}_{n}^{1}(k)$ the group of all $k$-algebra automorphisms $f$ on $k^{[[n]]}$ satisfying $f \equiv \operatorname{Id~} \bmod \left(I^{2}\right)$. Suppose $q \geq 1$ and $r \geq 1$. Let $G \subset \widehat{G A}_{1}^{1}(k)$ be the subset defined by $\left\{f: f(x)=x+c_{1} x^{q+1}+\cdots+c_{i} x^{i q+1}+\cdots\right.$, with $\left.c_{i} \in k\right\}$. It is easy to see that $G$ is closed under composition and inverses, and is thus a subgroup. Let $G_{r}$ be the group of all $k$-algebra automorphisms $f$ on $k^{[[1]]} /(x)^{r q+2}$ for which $f(x)=x+c_{1} x^{q+1}+\cdots+c_{r} x^{r q+1}$.

Suppose $N$ is an $n \times n$ matrix over $k^{[n]}$ with $N^{i}$ exact for all $i \geq 1$. (Actually the exactness of $N$ and $N^{2}$ implies exactness for $N^{i}$ for all $i \geq 2$. See note at the end of this paper.) Suppose also that each term of $N$ is a homogeneous polynomial of degree $q$. Let $U_{j}$ be the column vector

$$
U_{j}=\left(\begin{array}{c}
u_{1, j} \\
\vdots \\
u_{n, j}
\end{array}\right)
$$

with $J\left(U_{j}\right)=\left((j q+1) /(q+1)^{j}\right) N^{j}$ and with each $u_{i, j}$ a homogeneous polynomial of degree $j q+1$. (Note that if $n=1$, these conditions are satisfied if $U_{j}=x^{j q+1}$ and $N=(q+1) x^{q}$. Thus all relations developed for the $U_{j}$ will, in particular, apply to this special case.) Define a subset $H \subset \widehat{G A}_{n}^{1}(k)$ by $H=\{g: g(X)=$ $X+c_{1} U_{1}+\cdots+c_{i} U_{i}+\cdots$, with $\left.c_{j} \in k\right\}$. Here $g(X)$ represents the vector whose $i$ th term is $g\left(x_{i}\right)$.

Let $H_{r}$ be the set of all $k$-algebra automorphisms $g$ on $k^{[[n]]} / I^{r q+2}$ with $g(X)=X+c_{1} U_{1}+\cdots+c_{r} U_{r}$.

There are natural surjective maps $\alpha: G \rightarrow H$ and $\alpha_{r}: G_{r} \rightarrow H_{r}$. If $f \in G$ has $f(x)=x+c_{1} x^{q+1}+c_{2} x^{2 q+1}+\cdots$, then $g=\alpha(f)$ has $g(X)=X+c_{1} U_{1}+c_{2} U_{2}+\cdots$. The results of this paper are summarized in the following theorem.

Theorem. (i) Each of $H$ and $H_{r}$ is a group under composition. The natural surjective maps $\alpha: G \rightarrow H$ and $\alpha_{r}: G_{r} \rightarrow H_{r}$ are group homomorphisms. If $N^{i}$ is nonzero for all $i \geq 1$, then each of $\alpha$ and $\alpha_{r}$ is an isomorphism.

(ii) Suppose $N^{r+1}$ is zero. Then $H$ and $H_{r}$ are isomorphic. If $f \in H$, then each of $f$ and $f^{-1}$ maps $k^{[n]}$ into itself, and thus 
$f$ defines an automorphism on $k^{[n]}$. Thus $H$ may be viewed as a group of automorphisms on $k^{[n]}$. Finally, if $N^{r}$ is nonzero, then $\alpha_{r}: G_{r} \rightarrow H_{r}$ is an isomorphism.

(iii) Suppose $N^{r+1}$ is zero, $1 \leq s \leq r, 1 \leq t \leq r$, and $s+t>r$. Suppose $f, g \in H$ with $f(X)=X+c_{1} U_{1}+\cdots+c_{r} U_{r}$ and $g(X)=$ $X+d_{1} U_{1}+\cdots+d_{r} U_{r}$, with $c_{i}=0$ for $1 \leq i<s$ and $d_{i}=0$ for $1 \leq i<t$. Then $f \circ g(X)=g \circ f(X)=X+b_{1} U_{1}+\cdots+b_{r} U_{r}$ where $b_{i}=c_{i}+d_{i}$. Also $\left.J(f)\right|_{g}=J(f),\left.J(g)\right|_{f}=J(g)$, and $J(f \circ g)=J(f) J(g)=J(g) J(f)$.

Sketch of proof. Part (ii) follows immediately from part (i). The equalities stated in (iii) follow from inspection in the case where $f, g \in G_{r}$. Thus part (iii) follows from part (i) and (ii). We now sketch the proof of part (i).

Let $\mathscr{L}\left(\widehat{G A}_{n}^{1}(k)\right)$ be the Lie algebra of $\widehat{G A}_{n}^{1}(k)$. This is the $k$-module of all $k$-derivations $D$ on $k^{[[n]]}$ with $D\left(x_{i}\right) \in I^{2}$ for $1 \leq i \leq n$. Each $D$ is represented by a column vector

$$
V=\left(\begin{array}{c}
v_{1} \\
\vdots \\
v_{n}
\end{array}\right)
$$

with $D=v_{1}\left(\partial / \partial x_{1}\right)+\cdots+v_{n}\left(\partial / \partial x_{n}\right)$, where each $v_{i} \in k^{[[n]]}$ is the sum of homogeneous polynomials of degree $\geq 2$. If $W$ is any column vector, $D(W)$ is the column vector whose $i$ th term is $D\left(w_{i}\right)$. Thus $D(W)=J(W) V$. If $D_{1}$ and $D_{2}$ are derivations, then $\left[D_{1}, D_{2}\right]$ is the derivation $D_{1} D_{2}-D_{2} D_{1}$.

There is a bijection Exp: $\mathscr{L}\left(\widehat{G A}_{n}^{1}(k)\right) \rightarrow \widehat{G A}_{n}^{1}(k)$ given by $\operatorname{Exp}(D)(h)=e^{D}(h)=h+D(h)+\left(\frac{1}{2}\right) D^{2}(h)+\cdots$. The inverse of this bijection is given by $\log (f)=(f-I)-\left(\frac{1}{2}\right)(f-I)^{2}+\left(\frac{1}{3}\right)(f-I)^{3} \cdots$.

For the case $n=1$, the Lie algebra of $G$ is

$$
\mathscr{L}(G)=\left\{\left(d_{1} x^{q+1}+\cdots+d_{i} x^{i q+1}+\cdots\right) \frac{\partial}{\partial x}: d_{i} \varepsilon k\right\} .
$$

It is slightly tedious but straightforward to show that $G$ is the image of $\mathscr{L}(G)$ under the Exp map. For the general case, define $L \subset \mathscr{L}\left(\widehat{G A}_{n}^{1}(k)\right)$ to be all the derivations represented by vectors of the form $d_{1} U_{1}+\cdots+d_{i} U_{i}+\cdots$. There is a natural surjection $\beta: \mathscr{L}(G) \rightarrow L$ which sends $\left(d_{1} x^{q+1}+\cdots+d_{i} x^{i q+1}+\cdots\right)(\partial / \partial x)$ to the derivation represented by the vector $\left(d_{1} U_{1}+\cdots+d_{i} U_{i}+\cdots\right)$. 
It will follow from the first four steps below that $L$ is a Lie subalgebra of $\mathscr{L}\left(\widehat{G A}_{n}^{1}(k)\right)$ and that $\beta$ is a Lie algebra homomorphism from $\mathscr{L}(G)$ onto $L$. It will then be observed that $\operatorname{Exp} \circ \beta=\alpha \circ \operatorname{Exp}: \mathscr{L}(G) \rightarrow H$ and thus $H$ is the image of $L$ under the Exp map. In other words, the following diagram is commutative:

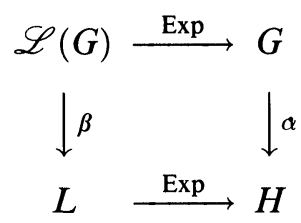

Since $L$ is a Lie subalgebra and $\beta$ is a Lie algebra homomorphism, it follows that $H$ is a subgroup of $\widehat{G A}_{n}^{1}(k)$ and that $\alpha$ is a group homomorphism.

Step 1: $U_{j}=(1 /(j q+1)) J\left(U_{j}\right) X=\left(1 /(q+1)^{j}\right) J\left(U_{1}\right)^{j} X$. The first equality is Euler's formula and the second equality follows from the equations $J\left(U_{1}\right)=N$ and $J\left(U_{j}\right)=\left((j q+1) /(q+1)^{j}\right) N^{j}$.

Step 2: $J\left(U_{i}\right) U_{j}=\left((i q+1) /(q+1)^{i}\right) J\left(U_{1}\right)^{i}\left(1 /(q+1)^{j}\right) J\left(U_{1}\right)^{j} X$ $=\left((i q+1) /(q+1)^{i+j}\right) J\left(U_{1}\right)^{i+j} X=(i q+1) U_{i+j}$. These equations follow from Step 1.

Step 3: Let $E_{s}=u_{1, s}\left(\partial / \partial x_{1}\right)+\cdots+u_{n, s}\left(\partial / \partial x_{n}\right)$, i.e., $E_{s}$ is the derivation represented by $U_{s}$. Then $\left[E_{i}, E_{j}\right]$ is represented by $J\left(U_{j}\right) U_{i}-J\left(U_{i}\right) U_{j}=(j q+1) U_{i+j}-(i q+1) U_{i+j}=(j-i) q U_{i+j}$. Thus $\left[E_{i}, E_{j}\right]=(j-i) q E_{i+j}$. Since any $E \in L$ can be written as $E=d_{1} E_{1}+d_{2} E_{2}+\cdots$, it follows that $L$ is a Lie subalgebra.

Step 4: $\beta$ is a Lie algebra homomorphism. First apply Step 3 to the case $n=1$ and $U_{i}=x^{i q+1}$. Let $D_{s}=x^{s q+1}(\partial / \partial x)$. Then by Step 3, $\left[D_{i}, D_{j}\right]=(j-i) q D_{i+j}$. Now $\beta\left(D_{s}\right)=E_{s}$ and so $\beta\left(\left[D_{i}, D_{j}\right]\right)=\beta\left((j-i) q D_{i+j}\right)=(j-i) q E_{i+j}=\left[E_{i}, E_{j}\right]=$ $\left[\beta\left(D_{i}\right), \beta\left(D_{j}\right)\right]$. Now any $D \in \mathscr{L}(G)$ can be written as $D=$ $d_{1} D_{1}+d_{2} D_{2}+\cdots$. Thus $\beta$ is a Lie algebra homomorphism.

Step 5: $\alpha \circ \operatorname{Exp}=\operatorname{Exp} \circ \beta: \mathscr{L}(G) \rightarrow \widehat{G A}_{n}^{1}(k)$. This is somewhat tedious to prove, but intuitively obvious, because the formula in Step 2 does not depend upon $n$.

Step 6: $H=\operatorname{Exp}(L)$ because $H=\alpha(G)=\alpha(\operatorname{Exp}(\mathscr{L}(G)))=$ $\operatorname{Exp}(\beta(\mathscr{L}(G)))=\operatorname{Exp}(L)$. Thus $H$ is a group and $L$ is the Lie algebra of $H, L=\mathscr{L}(H)$. Furthermore, since $\beta: \mathscr{L}(G) \rightarrow \mathscr{L}(H)$ is a Lie algebra homomorphism, $\alpha: G \rightarrow H$ is a group homomor- 
phism. (See [Bo, Chapter 2], [P, Section 9].) This completes the proof.

Final note. In the hypothesis of the main theorem, $N^{i}$ is exact for all $i \geq 1$. It suffices to assume only that $N$ and $N^{2}$ are exact, as shown by the following theorem.

Theorem. Suppose $M$ is an $n \times n$ matrix over $k^{[n]}$ and $M$ and $M^{2}$ are exact. Then for each $i \geq 1, M^{i}$ is exact. Furthermore, if $M$ is invertible, then for each integer $i, M^{i}$ is exact.

This is a special case of a general theory of closed, exact, and coexact matrices (see $[\mathrm{CZ}]$ ).

\section{REFERENCES}

[A] D. J. Anick, Limits of tame automorphisms of $k\left[x_{1}, \ldots, x_{n}\right]$, J. Algebra 82 (1983), 459-468.

[B] H. Bass, Algebraic K-theory, Benjamin, New York, 1968.

[BCW] H. Bass, E. H. Connell, and D. L. Wright, The Jacobian conjecture: reduction of degree and formal expansion of the inverse, Bull. Amer. Math. Soc. 7 (1982), 287-329.

[Bo] N. Bourbaki, Lie groups and Lie algebras, Hermann, Paris, 1971.

[C] E. H. Connell, On the $K$-theory of algebras and polynomial extensions , J. Pure Appl. Algebra 7 (1976), 169-174.

[CZ] E. Connell and J. Zweibel, Exact and coexact matrices, J. Algebra (to appear).

[P] M. Pittaluga, On the automorphism group of a polynomial algebra, thesis, Columbia University, 1984.

[W] S. Wang, A Jacobian criterion for separability, J. Algebra 65 (1980), 453494.

Department of Mathematics and Computer Science, University of Miami, Coral Gables, Florida 33124

Department of Mathematics, Florida International University, MIAMI, FLORIDA 33199 